\title{
Influence of organic solvent on optical and structural properties of ultra-small silicon dots synthesized by UV laser ablation in liquid $\dagger$
}

\author{
Romuald Intartaglia, ${ }^{a}$ Komal Bagga ${ }^{a}$ Alessandro Genovese, ${ }^{b}$ \\ Athanassia Athanassiou, ${ }^{a c}$ Roberto Cingolani, ${ }^{a}$ Alberto Diaspro ${ }^{a}$ and \\ Fernando Brandi ${ }^{a}$
}

Received 29th June 2012, Accepted 20th September 2012

DOI: $10.1039 / \mathrm{c} 2 \mathrm{cp} 42195 \mathrm{j}$

Ultra small silicon nanoparticles (Si-NPs) with narrow size distribution are prepared in a one step process by UV picosecond laser ablation of silicon bulk in liquid. Characterization by electron microscopy and absorption spectroscopy proves Si-NPs generation with an average size of $2 \mathrm{~nm}$ resulting from an in situ photofragmentation effect. In this context, the current work aims to explore the liquid medium (water and toluene) effect on the Si-NPs structure and on the optical properties of the colloidal solution. Si-NPs with high pressure structure (s.g. Fm $3 m$ ) and diamond-like structure (s.g. $F d 3 m$ ), in water, and $\mathrm{SiC}$ moissanite $3 \mathrm{C}$ phase (s.g. $F \overline{4} 3 m$ ) in toluene are revealed by the means of High-Resolution TEM and HAADF-STEM measurements. Optical investigations show that water-synthesized Si-NPs have blue-green photoluminescence emission characterized by signal modulation at a frequency of $673 \mathrm{~cm}^{-1}$ related to electron-phonon coupling. The synthesis in toluene leads to generation of Si-NPs embedded in the graphitic carbon-polymer composite which has intrinsic optical properties at the origin of the optical absorption and luminescence of the obtained colloidal solution.

\section{Introduction}

Luminescent ultra-small silicon nanoparticles (Si-NPs) with diameters in the range of $1-3 \mathrm{~nm}$ have triggered large research activity with an outlook for various applications ranging from light-emitting devices ${ }^{1,2}$ and photovoltaic solar cell technology, ${ }^{3}$ to fluorescent biological label ${ }^{4,5}$ and therapy. ${ }^{6}$ Due to their biocompatibility, high brightness and stability against photobleaching, Si-NPs are considered to be ideal candidates for substituting the fluorescent dyes in bio-tagging and bio imaging. ${ }^{7,8}$ They can also be integrated within the existing silicon technologies for creating nanoscale optoelectronic devices. ${ }^{3} \mathrm{Up}$ to now, several chemical $^{9,10}$ and physical methods ${ }^{11}$ have been developed for the Si-NPs synthesis. Compared to the physical method, the solution chemistry route presents the advantages of controlling simultaneously size and surface chemistry of NPs which is required for NP functionalisation. However, such techniques have the drawback of involving the use of chemical products and reducing agents or require multi-step procedures for the NPs size control. ${ }^{9}$ Pulsed laser ablation in liquid (PLAL) is an

\footnotetext{
${ }^{a}$ Nanophysics, Istituto Italiano di Tecnologia, via Morego, 30, 16163 Genova, Italy. E-mail: romuald.intartaglia@iit.it

${ }^{b}$ Nanochemistry, Istituto Italiano di Tecnologia, via Morego, 30, 16163 Genova, Italy

${ }^{c}$ Center for Biomolecular Nanotechnologies@UniLe, Istituto Italiano di Tecnologia, via Barsanti, 73010 Arnesano, Lecce, Italy

$\dagger$ Electronic supplementary information (ESI) available. See DOI $10.1039 / \mathrm{c} 2 \mathrm{cp} 42195 \mathrm{j}$
}

alternative approach for the fabrication of silicon nanoparticles. ${ }^{12-16}$ PLAL has some advantages: (i) a clean synthesis, without the requirement of chemical precursors; (ii) simple, since it is performed under ambient conditions; (iii) versatile, since the obtained NPs are in colloidal solution form, giving the opportunity for further nanoscale manipulations, such as biofunctionalization. ${ }^{17}$

Photoluminescence (PL) properties of Si-NPs produced by PLAL in various solvents have been reported. Svrcek et al. have shown that the PL of Si-NPs obtained in water is located near $400 \mathrm{~nm}^{14}$ Lu et al. reported that the luminescence of Si-NPs in toluene is in the visible range around $450 \mathrm{~nm} .{ }^{15}$ Recently, luminescence measurements on Si-NPs prepared by PLAL in ethanol displayed PL peak feature at around $630 \mathrm{~nm} .{ }^{16}$ Specifically, it was reported that similarity between PL of Si-NPs prepared in ethanol with mean size between 2 and $3 \mathrm{~nm}$ and with mean size between 5 and $10 \mathrm{~nm}$ suggests a common origin, which is probably not due to the pure size effect, i.e. quantum confinement. These previous works confirm that the liquid media in PLAL experiments have a strong influence on the optical properties of the obtained colloidal solution. As a consequence, further investigations of the optical properties of the colloidal solutions obtained by PLAL of silicon in organic solvent are necessary in order to clarify a situation with very high technological potentialities.

The production of small Si-NPs via PLAL has been investigated for different pulse durations and laser wavelengths. Most of the studies report synthesis of small Si-NPs via the PLAL method using nanosecond laser pulses, resulting in NPs 
which stabilize into clusters. ${ }^{13,14}$ Post-chemical treatment of the clusters is then required since most of the applications need non-agglomerated nanoparticles. ${ }^{13}$ Recently, our group investigated the generation of Si-NPs by near-infrared femtosecond PLAL demonstrating a size control mechanism dependent on the laser pulse energy. ${ }^{12}$ On the other hand, a widely studied method to reduce the size of NPs, especially metallic, is the photofragmentation, i.e., the fragmentation of large NPs into small NPs by laser irradiation of the colloidal solution. NPs photofragmentation has been investigated either ex-situ, ${ }^{18-21}$ i.e., by irradiation of the NPs colloidal solution or in situ, ${ }^{22-24}$ i.e., during NPs generation by PLAL.

Here, the UV picosecond laser ablation method is shown to be a simple technique to prepare ultra-small and stable Si-NPs with narrow size distribution in deionized water and toluene. Characterization by Transmission Electron Microscopy (TEM) and absorption spectroscopy reveals the generation of Si-NPs with an average size of $2 \mathrm{~nm}$. The nanoparticle size results from the size-quenching effect due to an in situ photofragmentation process. In this context, the current work aims to explore the liquid medium effect on the silicon nanoparticle structure and on the optical properties of the colloidal solution. Si-NPs with high pressure structure (s.g. Fm3m) and diamond-like structure (s.g. $F d 3 m$ ), in water, and also the $\mathrm{SiC}$ moissanite $3 \mathrm{C}$ phase (s.g. $F \overline{4} 3 m$ ) in toluene are revealed by the means of High-Resolution TEM and High Angular Annular Dark Field Scanning TEM measurements (HAADF-STEM), Energy Dispersive X-Ray Spectroscopy. Optical analysis shows that Si-NPs synthesized in water have blue-green photoluminescence emission, characterized by signal modulation at a frequency of $673 \mathrm{~cm}^{-1}$ related to electron-phonon coupling. In the case of toluene liquid, our experimental approach, consisting of the comparison of the optical properties (absorption and PL) between the colloidal solution obtained by PLAL and by the irradiated toluene solvent without the silicon target, revealed that pulsed UV irradiation of toluene generates a carbon based byproduct (graphitic carbon-polymer composite) having staggering optical properties matching with the ones of the colloidal solution obtained by PLAL of silicon. This allows for the first time the clarification of the origin of the optical properties of the colloidal solution obtained by UV PLAL of silicon in toluene.

\section{Experimental methods}

\subsection{Synthesis of Si nanoparticles}

Synthesis of Si-NPs was carried out using a Continuum Leopard laser providing pulses at $355 \mathrm{~nm}$, with a time duration of 60 picosecond, and at a repetition rate of $20 \mathrm{~Hz}$. The laser beam with a diameter of $6 \mathrm{~mm}$ is focused $10 \mathrm{~mm}$ below the target surface using a lens with a focal length of $30 \mathrm{~cm}$. Pulse energy was fixed at $1 \mathrm{~mJ}$. The silicon target (99.999\% from Alpha Aesar), in the form of a cylinder with a diameter of $6 \mathrm{~mm}$ and a thickness of $10 \mathrm{~mm}$, was placed on the bottom of a quartz cuvette (dimension $10 \times 10 \times 30 \mathrm{~mm}^{3}$ ) and immersed in $2 \mathrm{ml}$ of liquid medium, either deionized water or toluene (toluene spectrometric grade from Aldrich). The height of the liquid above the target surface was $16 \mathrm{~mm}$. Before each experiment the target was mechanically polished and then washed with the same liquid used for the ablation several times to remove impurity from the surface. During laser ablation, the target was moved using a rotation system to achieve more uniform irradiation of the silicon surface. After 60 min of ablation, the solution was transparent for deionized water, while it became brown for toluene solvent. As a control test, both solvents were also irradiated without the presence of a target, under the identical experimental conditions shown in Fig. S1 (ESI $\dagger)$. The toluene solvent became brown, while water did not change colour, as expected. All characterization measurements were performed 1 day after preparation of the colloidal solution.

\subsection{Characterization}

The Si-NPs colloidal solutions were deposited directly onto the carbon-coated 300 mesh copper grid and were characterized by Conventional TEM, High resolution TEM and High Angular Annular Dark Field (HAADF) Scanning TEM (STEM) measurements performed with a JEOL JEM-2200FS microscope, equipped with a field emission gun working at an accelerating voltage of $200 \mathrm{kV}$, a CEOS spherical aberration corrector of the objective lens allowing to reach a spatial resolution of $0.9 \AA$, and an in-column Omega filter. Chemical compositions were determined for several NPs by Energy Dispersive X-ray Spectroscopy (EDS) analysis performed in the STEM mode, using a JED-2300 Si(Li) detector and using an electron probe of $0.7 \AA$ size. A 2 ml colloidal solution was used to measure the absorption and photoluminescence spectra. Optical absorption spectra were recorded in a quartz cuvette (10 mm, Helma), using a cary $6000 \mathrm{UV}$-VIS double beam spectrophotometer. The scan range was $200-600 \mathrm{~nm}$ at a $600 \mathrm{~nm} \mathrm{~min}{ }^{-1}$ rate. The photoluminescence (PL) measurements for colloidal solution were carried out in the $350-600 \mathrm{~nm}$ wavelength range using a Fluoroma-4 Spectrofluorometer (JobinYvon-Horiba) fitted with a photomultiplier and a Xenon lamp source. PL and absorption spectra were corrected for solvent contribution.

\section{Results and discussion}

\subsection{Water-synthesised Si-NPs}

The generation of Si-NPs by UV picosecond laser ablation was investigated by systematic studies of the Si-NPs colloidal solution produced at different irradiation times. Detailed information about the morphology and size distribution of the Si-NPs in water was obtained by TEM measurements. Fig. 1 shows the TEM image of the Si-NPs obtained after (a) $5 \mathrm{~min}$ and (b) $60 \mathrm{~min}$ of irradiation. For short ablation time, the majority of the Si-NPs produced have an average size of $10 \mathrm{~nm}$ as displayed in Fig. 1a. At longer irradiation time, $60 \mathrm{~min}$, isolated Si-NPs i.e., non-agglomerated matrix free NPs, with a spherical morphology are observed. The Si-NPs size distribution, shown in the inset of the microphotograph, was obtained considering more than 500 particles. The vast majority of the produced NPs have very small size (average size of $\sim 2 \mathrm{~nm}$ ) but rarely larger NPs are also observed. From TEM analysis of a bigger area $(500$ micrometer $\times 500$ micrometer, 


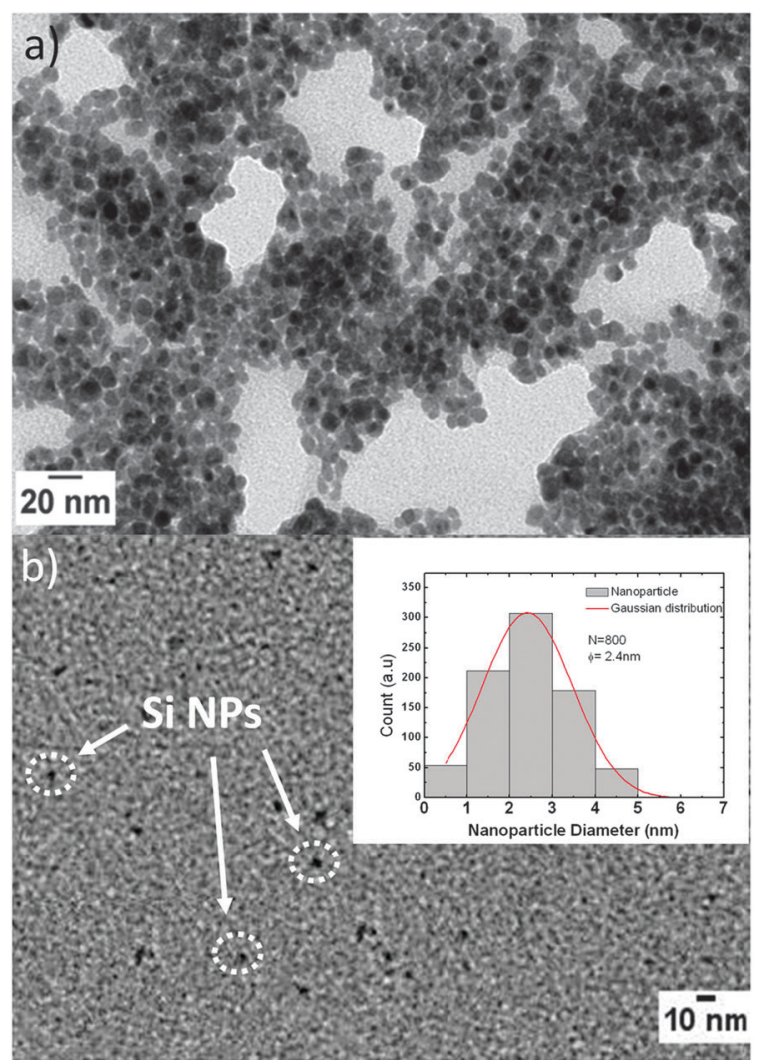

Fig. 1 TEM images of Si-NPs synthesized in water via UV PLAL after (a) $5 \mathrm{~min}$ and (b) $60 \mathrm{~min}$ of irradiation.

not reported), we can estimate that less than 1 percent of larger NPs are present. The nanoparticle size reduction is attributed to the well-known photofragmentation effect. ${ }^{22,24}$ The photofragmentation process is responsible also for the change in color of the solution over time, varying from light yellow for large size NPs with an absorption band up to $470 \mathrm{~nm}$ to completely transparent for smaller size NPs with an absorption band below $300 \mathrm{~nm} .{ }^{12}$

Complementary to TEM analysis, HAADF-STEM, EDX-STEM and HRTEM measurements were carried out to determine the chemical composition and the crystalline structure of the NPs. The HAADF-STEM image, presented in Fig. 2a, shows the presence of ultra-small Si-NPs together with a $\mathrm{Si}$ single particle (Si-SP) having a diameter of about $100 \mathrm{~nm}$. Fig. 2b reports the EDX spectra of Si-SP and Si-NPs, respectively, obtained by scanning the electron probe over two regions of equal area. Both spectra reveal only the Si K $\alpha$ peak, but with different intensity because the signal of Si-NPs is generated from a small scattering-volume with respect to that of Si-SP. Fig. 2c shows the HRTEM images of Si-NPs having diamond-like crystal structure $(F d 3 m)$ with the typical (111) lattice sets and a $d$-spacing of $3.12 \AA$ (Fig. 2c). Other crystallites (Fig. 2d) exhibit lattice parameters and numerical electron diffraction patterns not consistent with the diamond-like silicon, but compatible with a cubic silicon phase with space group $F m 3 m$ characterized by smaller lattice parameters, and are stable in high pressure, 80 kbar (PDF card 35-1158). This is due to the conditions far from the equilibrium at the local level provided by pulsed laser ablation in solution. ${ }^{25}$

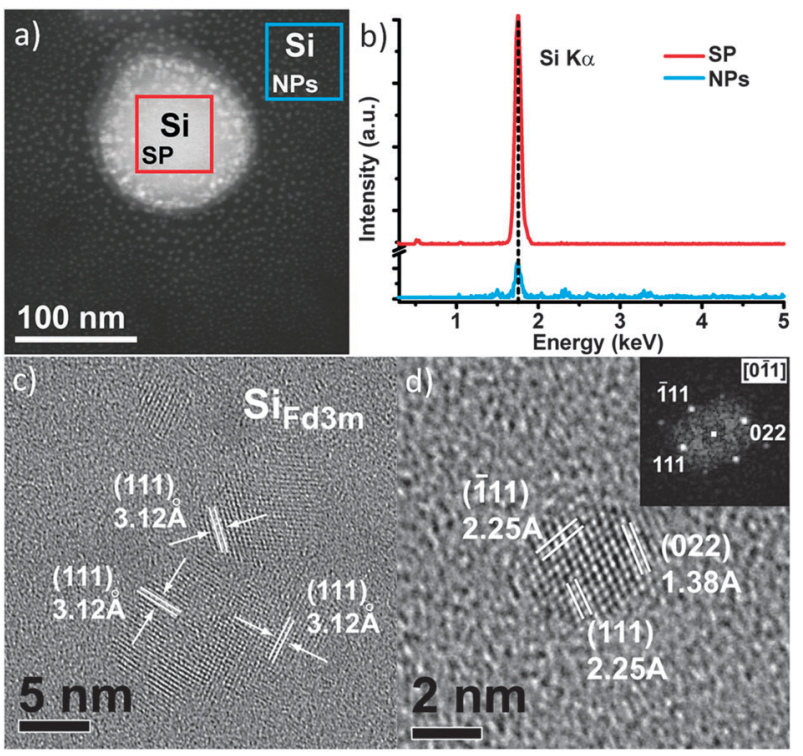

Fig. 2 (a) HAADF STEM image displaying both a bigger single particle Si-SP and ultra small Si-NPs synthesized in water, red and cyan squares highlight, respectively, the two regions scanned for the areal STEM-EDX analyses, and (b) corresponding EDX spectra, red for the Si-SP and cyan for the Si-NPs, showing only the presence of $\mathrm{Si}$ $\mathrm{K} \alpha$ peaks. HRTEM images of obtained Si-NPs via UV picosecond laser ablation in water. (c) Single Si-NP synthesized in water showing the (111) lattice sets with a $d$-spacing of $3.12 \AA$, characteristic of silicon with $F d 3 m$ structure. (d) Single Si-NP synthesized in water showing the (111) and (022) lattice sets with $d$-spacings of $2.25 \AA$ and $1.38 \AA$, respectively, the numerical electron diffraction pattern (inset) is compatible with the $\left[\begin{array}{lll}0 & \overline{1} & 1\end{array}\right]$ zone axis projection of $F m 3 m$ structure.

Due to the large difference in temperature $(10000 \mathrm{~K})$ and pressure (about $1 \mathrm{GPa}$ ) between the hot plasma and the surrounding medium, the growth of Si-NPs takes place inside a high pressure cavitation bubble, leading to the formation of cubic silicon phase.

Optical properties of Si-NPs synthesized in water after $60 \mathrm{~min}$ of irradiation are reported in Fig. 3. A strong absorption band in the UV range (black line) is observed corresponding to a population with a size less than $3 \mathrm{~nm}^{12}$ in agreement with the corresponding electron micrograph analysis (Fig. 1b). Due to the presence of NPs with size smaller than the Bohr exciton radius in bulk materials, PL emission of water-synthesized Si-NPs was observed. The PL spectrum shows peak emission in the blue-green range at $460 \mathrm{~nm}$, characterized by discrete optical transitions. Theoretical and experimental investigation relates the vibrational structure of photoluminescence i.e. discrete transition, to the chemical nature of the NP surface. For surface oxidized (carbide) Si-NPs, electron-photon coupling between excitons and phonons of $\mathrm{Si}-\mathrm{O}-\mathrm{Si}(\mathrm{Si}-\mathrm{C})$ vibration generates features in the emission spectra with a constant spacing of $0.13-0.17 \mathrm{eV}(0.18 \mathrm{eV}){ }^{26-29}$

In the present case, the constant spacing between the discrete structures is around $0.083 \mathrm{eV}$, which corresponds to $673 \mathrm{~cm}^{-1}$, and it is attributed to the $\mathrm{Si}-\mathrm{O}$ bending vibrations mode. ${ }^{30}$ During the laser ablation process, the decomposition of water molecules into $\mathrm{O}$ and $\mathrm{OH}^{-}$radicals occurs via thermolysis due to extreme local temperature ${ }^{31}$ as well as via 


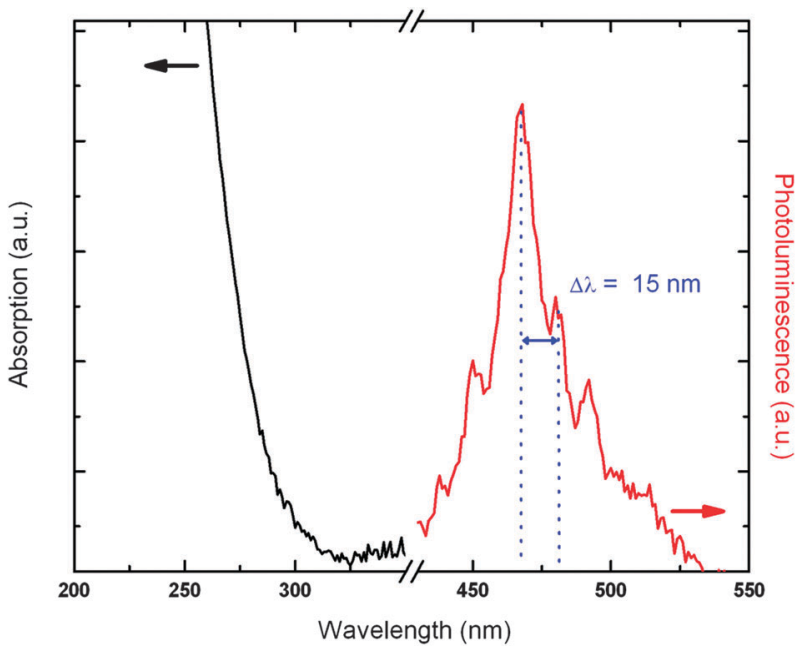

Fig. 3 Optical properties of the Si NPs colloidal solution via UV picosecond laser ablation in water after $60 \mathrm{~min}$ of irradiation, absorption spectrum (black line) and PL spectrum (red line). Excitation wavelength used for PL measurement: $290 \mathrm{~nm}$.

photolysis due to near UV irradiation through two-photon absorption. $^{32}$

\subsection{Toluene-synthesised Si-NPs}

Laser ablation of the silicon target in toluene solvent is investigated in order to clarify the effects on the structural and optical properties of the resulting colloidal solution, e.g. strong photoluminescence response as that reported in ref. 15. Fig. 4a shows a TEM image of the Si-NPs synthesized in toluene via PLAL after $60 \mathrm{~min}$ of UV irradiation under the same laser pulse energy conditions used for Si-NPs generation in water. Si-NPs are found to be ultra small with a mean size of around $2 \mathrm{~nm}$. HRTEM data analysis revealed the nucleation of rare Si-NPs exhibiting lattice parameters compatible with the Si moissanite $3 \mathrm{C}$ phase with space group $F \overline{4} 3 m$ (PDF card 75-0254). An example of single SiC NPs is shown in Fig. 4c where the lattice sets (111) and (220) of moissanite $3 \mathrm{C}$ are displayed. The formation of $\mathrm{SiC}-\mathrm{NPs}$ results from a decomposition of the solvent, similarly to what has been found by PLAL of silicon in ethanol solvent. ${ }^{16}$ Moreover, some crystallites synthesized in toluene exhibited lattice parameters and numerical electron diffraction patterns compatible with a cubic silicon phase with space group $F m 3 m$ characterized by smaller lattice parameters, and stable under high pressure conditions, 80 kbar (PDF card 35-1158) (Fig. 4b), as observed for $\mathrm{Si}-\mathrm{NPs}$ generated in water.

In contrast to the case of the PLAL in water, a black solid film surrounding Si-NPs is also observed. The generation of NPs embedded in the carbon byproduct and carbide compound via PLAL has been previously reported. ${ }^{33-35}$ The formation of carbon byproduct has been attributed to a reaction between the solvent molecules with the plasma plume during the ablation process. ${ }^{34}$ In particular, Yang et al. ${ }^{35}$ have reported the generation of carbon byproduct by laser ablation of the silicon target in toluene solvent, as revealed after posttreatment of the colloidal solution by centrifugation in order to remove the Si-NPs. The resulting supernatant, i.e., carbon

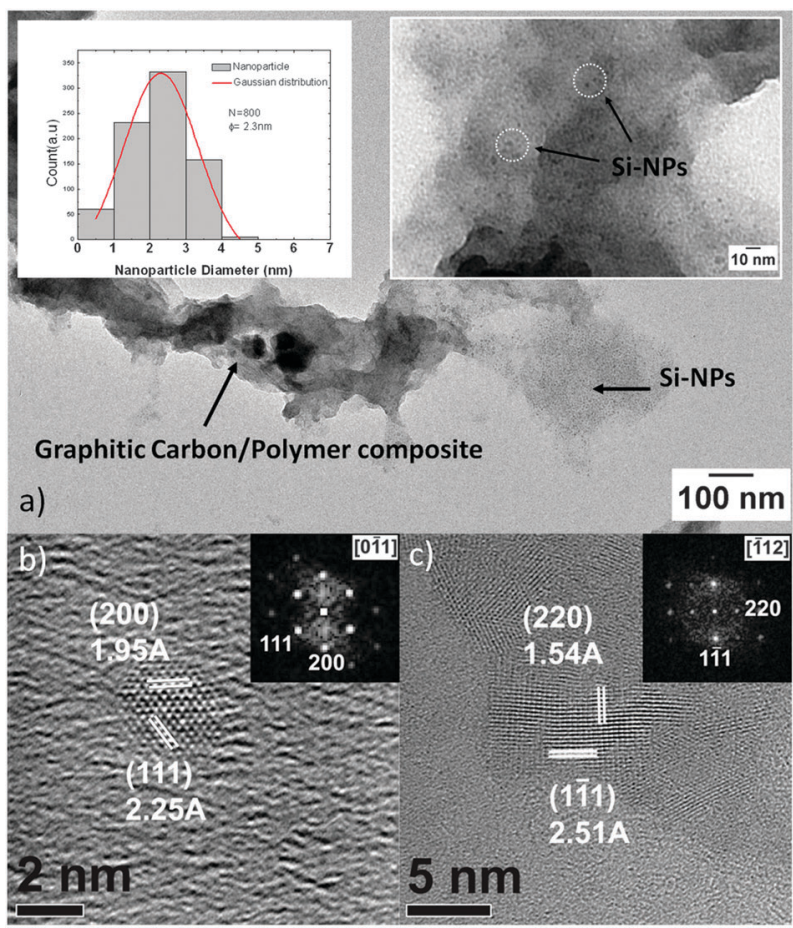

Fig. 4 (a) TEM image of the Si-NPs colloidal solution obtained via PLAL in toluene. Solid black film corresponds to the graphitic carbon-polymer composite, generated by photodecomposition of the toluene solvent. (b) HRTEM image of single Si-NPs showing the (111) and (200) lattice sets with $d$-spacings of $2.25 \AA$ and $1.95 \AA$, respectively, the numerical electron diffraction pattern (inset) is compatible with the $\left[\begin{array}{lll}0 & \overline{1} & 1\end{array}\right]$ zone axis projection of $F m 3 m$ structure. (c) HRTEM image of a single moissanite $3 \mathrm{C}$ crystal observed along its [lll 112$]$ zone axis, as confirmed by the numerical electron diffraction pattern (inset), and exhibiting the typical lattice sets (1 $1 \overline{1} 1)$ and (220) with $d$-spacings of

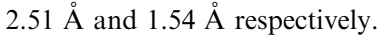

byproduct colloidal solution, has been shown to have luminescence properties. Similarly, it has been reported ${ }^{15}$ that the colloidal Si-NPs solution obtained via PLAL in toluene has emission properties similar to the supernatant solution reported in ref. 35. However, all these observations still lack control experiments, i.e., the irradiation of the solvent without the target under the same experimental conditions used for PLAL experiments, in order to better understand the process underlining the production of carbon byproduct.

We did perform the control experiment with the toluene solvent, as reported in the schematic of Fig. S1 (ESI $\dagger$ ). The TEM image of the irradiated toluene solvent after $60 \mathrm{~min}$ of irradiation without the presence of a target is reported in Fig. S2 (ESI $\dagger)$. It shows the presence of a carbon byproduct film similar to the one shown in the electron micrograph of the colloidal solution obtained by PLAL of silicon in toluene (Fig. 4a). This indicates that the toluene molecules interact directly with the $355 \mathrm{~nm}$ laser wavelength during the irradiation process, resulting in a modification of the chemical/ molecular structure of the solvent. This photochemical process is based on photolysis of toluene molecules, and the nature of the products is strongly dependent on the experimental conditions (pressure/temperature) as well as on the laser wavelength. ${ }^{36}$ Photochemical reactions like $\mathrm{C}-\mathrm{H}, \mathrm{C}-\mathrm{C}$ fissions 

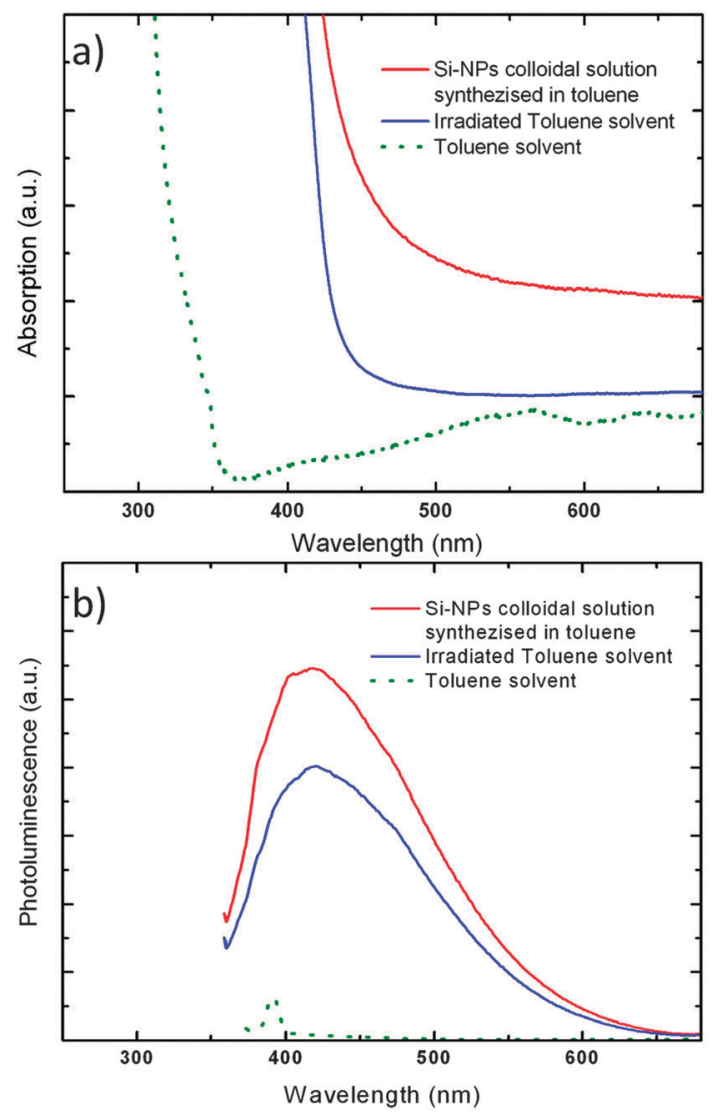

Fig. 5 (a) Absorption spectra of the fresh toluene solvent (dotted green line), UV irradiated toluene solvent (blue line) and Si NP colloidal solution synthesized in toluene (red line), after $60 \mathrm{~min}$ of irradiation. (b) PL spectra of the fresh toluene solvent (dotted green line), UV irradiated toluene solvent (blue line) and Si colloidal solution obtained via PLAL in toluene (excitation wavelength: $350 \mathrm{~nm}$ ).

take place during UV irradiation leading to the generation of graphitic carbon and polymeric $\mathrm{C} / \mathrm{H}$ agglomerates formation.

Optical properties of both carbon byproduct, generated by the irradiation of toluene solvent, and the colloidal solution obtained by PLAL of silicon in toluene were investigated and compared. Fig. 5a display the absorption spectra of the fresh toluene solvent (dotted green curve), UV irradiated toluene solvent (blue curve) and Si-NPs colloidal solution synthesized in toluene (red curve). Firstly, the absorption spectrum of the irradiated toluene solvent shows a clear red-shift of the absorption edge with respect to the one of the fresh toluene solvent. Thus, the carbon byproduct generated by UV irradiation of toluene solvent without the target has a distinct optical absorption spectrum with respect to the starting solvent. Secondly, Si-NPs colloidal solution synthesized in toluene displays an absorption edge with a cut-off in the same wavelength range as that for the irradiated toluene solvent (around $450 \mathrm{~nm}$ ).

Since the elements constituting the colloidal solution are the carbon byproduct and ultra small Si-NPs, the latter having an absorption cut-off of around $300 \mathrm{~nm}$ (Fig. 3), the observed absorption properties are attributed to the carbon byproduct. This proves that the absorption properties of Si-NPs colloidal solution synthesized in toluene arise from the carbon byproduct and not from Si-NPs. Further experimental works are in progress to give an insight into the molecular structure of the carbon byproduct obtained by $355 \mathrm{~nm}$ picosecond laser irradiation of toluene.

Fig. 5b shows PL spectra of the toluene solvent (doted green curve), UV irradiated toluene solvent (blue curve), and Si-NPs colloidal solution synthesized in toluene (red curve). No PL emission was observed for the non-irradiated toluene solvent (the small peak around $390 \mathrm{~nm}$ originating from Raman emission). The irradiated toluene solvent instead has PL emission in the visible range with a peak at around $420 \mathrm{~nm}$. Thus, the carbon byproduct generated by UV irradiation of toluene solvent has intrinsic luminescence properties. Si-NPs colloidal solution synthesised in toluene has a luminescence spectrum similar to the irradiated toluene solvent, only the PL intensity is slightly higher. This may indicate that silicon ablation plays a role of a catalyser increasing the amount of the carbon byproduct, and consequently the luminescence intensity. It is stressed that the perfect match found between the PL spectrum from colloidal solution obtained by PLAL of silicon in toluene and irradiated toluene explains the origin of previously reported luminescence properties. ${ }^{15}$

In summary, the mechanism behind the generation of carbon byproduct by PLAL in toluene solvent, which may nullify the inherent purity of the laser based method, is quite complex and needs to be investigated more in detail. It has been suggested that the carbon byproduct results from an interaction between solvent molecules and species present in the ablation plasma plume. ${ }^{34}$ Our approach, based on the control experiment without the target and on detailed optical investigations, reveals that other mechanisms have to be taken into account, such as direct interaction between solvent molecules and laser. This allows for the first time the distinction of the basic optical properties (absorption and PL) between the carbon byproduct and the generated Si-NPs constituting the colloidal solution in UV PLAL of silicon in toluene.

\section{Conclusion}

It is shown that UV picoseconds pulsed laser ablation of a solid target in liquid is a simple technique to synthesize luminescent ultra-small $(2-3 \mathrm{~nm})$ and well-dispersed Si-NPs. The obtained ultra small size results from an in-situ photofragmentation mechanism. Liquid media are found to influence the microstructure of the obtained Si-NPs and on the optical properties of the colloidal solution. Si-NPs with high pressure structure (s.g. $F m 3 m$ ) and diamond-like structure (s.g. $F d 3 m$ ) are observed for water. For toluene, besides Si-NPs, also SiC-NPs with moissanite $3 \mathrm{C}$ phase (s.g. $F \overline{4} 3 m$ ) are observed. PL studies show that the water-synthesized Si-NPs present very specific blue-green emission characterized by signal modulation at a frequency of $673 \mathrm{~cm}^{-1}$ related to electron-phonon coupling. Laser synthesis in toluene solvent leads to the formation of Si-NPs embedded in a graphitic carbon-polymer composite originating from the interaction of the toluene molecules with the UV photons. Detailed optical investigations demonstrate that the optical absorption and broad PL of toluene-synthesized Si-NPs obtained by PLAL arise from the graphitic carbonpolymer composite and not by the generated Si-NPs. 


\section{References}

1 R. Walters, H. Atwater and G. Bourianoff, Nat. Mater., 2005, 4, 143-146.

2 G. Belomoin, J. Therrien, A. Smith, S. Rao, R. Twesten, S. Chaieb, M. H. Nayfeh, L. Wagner and L. Mitas, Appl. Phys. Lett., 2002, 80, 841-843.

3 M. Stupca, M. Alsalhi, T. Al Saud, A. Almuhanna and M. H. Nayfeh, Appl. Phys. Lett., 2007, 91, 063107-063110.

4 F. Erogbogbo, K. T. Yong, I. Roy, R. Hu, W. C. Law, W. Zhao, H. Ding, F. Wu, R. Kumar, M. T. Swihart and P. N. Prasad, ACS Nano, 2011, 5, 413-423.

5 Z. F. Li and E. Ruckenstein, Nano Lett., 2004, 4, 8-13.

6 L. Xiao, L. Gu, S. B. Howell and M. J. Sailor, ACS Nano, 2011, 5, 3651-3659.

7 R. Intartaglia, K. Bagga, M. Scotto, A. Diaspro and F. Brandi, Opt. Mater. Express, 2012, 2, 510-518.

8 E. Borsella, M. Falconieri, N. Herlin, V. Loschenov, G. Miserocchi, Y. Nie, I. Rivolta, A. Ryabova and D. Wang, Biomedical and Sensor Applications of Silicon Nanoparticles, in Silicon Nanocrystals: Fundamentals, Synthesis and Applications, ed. L. Pavesi and R. Turan, Wiley-VCH Verlag GmbH \& Co. KGaA, Weinheim, Germany, 2010.

9 M. Rosso-Vasic, E. Spruijt, Z. Popovic, K. Overgaag, B. Van Lagen, B. Grandidier, D. Vanmaekelbergh, D. Dominguez-Gutierrez, L. De Cola and H. Zuilhof, J. Mater. Chem., 2009, 19, 5926-5933.

10 X. Zhang, D. Neiner, S. Wang, A. V. Louie and S. M. Kauzlarich, Nanotechnology, 2007, 18, 095601-095607.

11 J. Knipping, H. Wiggers, B. Rellinghaus, P. Roth, D. Konjhodzic and C. Meier, J. Nanosci. Nanotechnol., 2004, 4, 1039-1044.

12 R. Intartaglia, K. Bagga, F. Brandi, A. Genovese, E. Di Fabrizio and A. Diaspro, J. Phys. Chem. C, 2011, 115, 5102-5107.

13 K. Abderrafi, R. G. Calzada, M. B. Gongalsky, I. Suarez, R. Abarques, V. S. Chirvony, V. Y. Timoshenko, R. Ibanez and J. P. Martinez-Pastor, J. Phys. Chem. C, 2011, 115, 5147-5151.

14 V. Svrcek, D. Mariotti and M. Kondo, Opt. Express, 2009, 17, 520-527.

15 W. Lu, Y. Bian, H. Liu, L. Han, W. Yu and G. Fu, Mater. Lett., 2010, 64, 1073-1076.

16 P. G. Kuzmin, G. A. Shafeev, V. V. Bukin, S. V. Garnov, C. Farcau, R. Carles, B. Warot-Fontrose, V. Guieu and G. Viau, J. Phys. Chem. C, 2010, 114, 15266-15271.
17 R. Intartaglia, A. Barchanski, K. Bagga, A. Genovese, G. Das, P. Wagener, E. Di Fabrizio, A. Diaspro, F. Brandi and S. Barcikowski, Nanoscale, 2012, 4, 1271-1274.

18 F. Giammanco, E. Giorgetti, P. Marsili and A. Giusti, J. Phys. Chem. C, 2010, 114, 3354-3363.

19 D. Werner and S. Hashimoto, J. Phys. Chem. C, 2011, 115 5063-5072.

20 A. Pyatenko, M. Yamaguchi and M. Suzuki, J. Phys. Chem. C, 2009, 113, 9078-9085.

21 F. Mafune, J. Y. Kohno, Y. Takeda and T. Kondow, J. Phys. Chem. B, 2001, 105, 9050-9056.

22 A. Schwenke, P. Wagener, S. Nolte and S. Barcikowski, Appl. Phys. A: Mater. Sci. Process., 2011, 104, 77-82.

23 H. Muto, K. Miyajima and F. Mafune, J. Phys. Chem. C, 2008, 112, 5810-5815.

24 F. Mafune, J. Y. Kohno, Y. Takeda and T. Kondow, J. Phys. Chem. B, 2001, 105, 5114-5120.

25 K. Sasaki and N. Takada, Pure Appl. Chem., 2010, 82, 1317-1327.

26 J. Martin, F. Cichos, F. Huisken and C. Borczyskowski, Nano Lett., 2008, 8, 656-660.

27 J. D. Holmes, K. J. Ziegler, R. C. Doty, L. E. Pell, K. P. Johnston and B. A. Korgel, J. Am. Chem. Soc., 2001, 123, 3743-3748.

28 M. J. Llansola Portoles, F. R. Nieto, D. B. Soria, J. I. Amalvy, P. J. Peruzzo, D. O. Martire, M. Kotler, O. Holub and M. C. Gonzalez, J. Phys. Chem. C, 2009, 113, 13694-13702.

29 D. Tan, Z. Ma, B. Xu, Y. Dai, G. Ma, M. He, Z. Jinb and J. Qiu, Phys. Chem. Chem. Phys., 2011, 13, 20255-20261.

30 J. S. Bhaskar, G. Parthasarathy and N. C. Sarmah, Bull. Mater. Sci., 2008, 5, 775-779.

31 J. Lede, F. Lapicque and J. Villermaux, Int. J. Hydrogen Energy, 1983, 8, 675-679.

32 M. Ceppatelli, R. Bini and V. Schettino, PNAS, 2009, 106, 11454-11459.

33 V. Amendola, G. A. Rizzi, S. Polizzi and M. Meneghetti, J. Phys. Chem. B, 2005, 109, 23125-23128.

34 V. Amendola, P. Riello and M. Meneghetti, J. Phys. Chem. C, 2011, 115, 5140-5146.

35 S. Yang, H. Zeng, H. Zhao, H. Zhang and C. Weiping, J. Mater. Chem., 2011, 21, 4432-4436.

36 J. Pola, M. Urbanova, Z. Bastl, Z. Plzak, J. Subrt, V. Vorlicek, I. Gregorac, C. Crowley and R. Taylor, Carbon, 1997, 35, 605-611. 